\title{
Rasgos patológicos de personalidad y metabolitos cerebrales como predictores de no abstinencia en adictos con trastornos de personalidad
}

\section{Personality pathological traits and brain metabolites as predictors of non-abstinence in addicts with personality disorders}

\author{
| Daniel Serrani Azcurra*
}

| *Facultad de Psicología. Universidad Nacional de Rosario. Argentina

Enviar correspondencia a:
Daniel Serrani Azcurra,
Zeballos 1625,
danielserrani@argentina.com

\section{Resumen}

Se investigaron diferencias en rasgos de personalidad patológicos y alteraciones en metabolitos cerebrales entre no consumidores, consumidores abstinentes y no abstinentes. Los participantes $(n=113)$ entre $18-45$ años con trastorno de personalidad (TP) fueron diagnosticados con entrevistas clínicas y escalas para depresión, ansiedad, impulsividad y dimensiones de patologías de personalidad. Los metabolitos cerebrales se analizaron con resonancia magnética con espectroscopia. Los datos se analizaron con ANOVA y comparaciones múltiples. Abstinentes y no abstinentes se distinguieron de no consumidores por desregulación emocional, inhibición, y expresión restringida; abstinentes y no abstinentes se distinguieron entre si en auto-agresión, comportamiento disocial, problemas de conducta, búsqueda de estímulos y problemas de intimidad. Niveles de N-Acetil Aspartato y Creatina fueron menores para no abstinentes en cortezas prefrontal, cingulada anterior, vermis cerebeloso y corona radiada superior. Para abstinentes, niveles de colina fueron mayores en vermis cerebeloso y niveles de $n$-acetil aspartato fueron menores en corteza prefrontal dorsolateral, cingulada anterior e insula. En cuanto a rasgos de personalidad, apego inseguro, narcisismo, labilidad, autoagresión y ansiedad caracterizan a consumidores y abstinentes; en tanto suspicacia, rechazo y dureza de carácter se encuentran en consumidores (no abstinentes y abstinentes). Rasgos compulsivos, impulsión corporal no planeada, y descontrol en regulación de emociones predominaron en no abstinentes y participantes con co-morbilidades. Desapego e inhibición predominan en Trastorno por uso de Alcohol y rasgos narcisistas en uso de Sustancias.

Palabras claves: espectroscopia, personalidad, consumo de sustancias, abstinencia, no abstinencia, metabolitos cerebrales.

\begin{abstract}
Differences in pathological personality traits and disturbances in brain metabolites between non consumers, abstinent and non abstinent consumers were assessed. Participants $(n=113)$ aged between $18-45$ years with personality disorder (PD) were diagnosed with clinical interview and scales for depression, anxiety, impulsivity and dimensions of personality pathology. Brain metabolites were analyzed with magnetic resonance spectroscopy. Data were analyzed with ANOVA and multiple comparisons. Abstinent and non-abstinent differentiated from non-consumers in emotional deregulation, inhibition, and restricted expression; abstinent and non-abstinent differentiated from each other in self-aggression, dissocial behaviour, conduct disorder, stimulus seeking and intimacy problems. N-Acetyl Aspartate and creatine values were lower between non-abstinent in prefrontal, anterior cingulate cortex, cerebellar vermis and superior corona radiata. For abstinent, choline levels were greater in cerebellar vermis and $n$-acetyl aspartate were lower in dorso-lateral prefrontal and anterior cingulated cortex and insula. Regarding personality traits, insecure attachment, narcissism, lability, self-aggression and anxiety characterize consumers and abstinent, while suspiciousness, rejection and character hardness are found in consumers (non-abstinent and abstinent). Compulsive traits, unplanned body impulsiveness and lack of control in emotional regulation predominated in non-abstinent and participants with co-morbidities. Detachment and inhibition predominate in alcohol abuse disorder and narcissistic traits in substance abuse.
\end{abstract}

Key words: spectroscopy, personality, substance consumption, abstinence, non abstinence, brain metabolites. 
L os rasgos de personalidad patológicos se han vinculado con esquizoidia (Tibbo, 2004), esquizotipia (Downhill, 2001), personalidad obsesivo-compulsiva (Everitt, 2005, 2008; Bartha, 1998), evitativa y borderline (Van Elst, 2001), depresión (Hamakawa, 1998; Davanzo, 2003) y esquizofrenia (Buckley y Friedman, 2000; Bertolino, 2000; Dinzeo y Docherty, 2007). Sin embargo la asociación de rasgos patológicos de personalidad con abuso de sustancias genera desafíos (Ball, 2005); ya sea que los primeros se consideren como favorecedores de adicciones (Krueger y Tackett, 2003), o como una consecuencia de éstas (Herrero, 2004). Además, hay resultados favor de esta correlación (Elkins, 2006), y en contra (McGue, 1999), en ambos casos con limitado soporte empírico (Ball, 2006; Mateos y Mateos, 2005). Desinhibición, impulsividad, y manipulación predicen trastornos por uso de alcohol (TUA) y sustancias (TUS) en pacientes ambulatorios (Ready, 2006); pero no hay estudios que asocien rasgos patológicos de personalidad específicos con TUA, TUS, co-morbilidades, abstinentes en remisión, no abstinentes, 0 alteraciones neurobiológicas evaluadas con resonancia magnética espectroscópica. Los TUS y TUA se han vinculado con alteraciones del sistema de recompensa (Kalivas y Volkow, 2005; McFarland, 2003) especialmente corteza prefrontal medial (Pfleiderer, 2004), corteza cingulada y amígdala (Omura, 2005), vermis cerebeloso (Cecil et al, 2003), área tegmental ventral (Makris et al, 1999), vermis cerebeloso (Sullivan, 2003), corona radiada (Durazzo y Meyerhoff, 2007) y tractos de proyección fronto-parietales (Schlaepfer, 2006). Se ha descripto una asociación entre neuroticismo y extraversión con regiones (DeYoung,
2010) o volúmenes cerebrales. Por otra parte, la espectroscopía de resonancia magnética ( ${ }^{1} \mathrm{H}-\mathrm{MRS}$ ) permite medir neurometabolitos in vivo incluyendo $\mathrm{N}$-acetilaspartato (NAA), creatina (Cre) y colina (Co), cuyas alteraciones se han asociado con estados de ánimo (Jung, 2002), o creatividad (Jung, 2009). Con base en lo anterior, se formularon las siguientes hipótesis: a) TUS y TUA deberian asociarse con rasgos de personalidad desadaptativos y desinhibitorios, sobre todo en casos con co-morbilidad, b) TUS y TUA deberían correlacionarse con alteraciones neurometabólicas específicas, detectables con espectroscopía cerebral, contribuyendo a mantener o agravar el consumo o recaída. En consecuencia los propósitos de este estudio son investigar: a) rasgos de personalidad patológicos diferenciales para TUS y TUA abstinentes (remitidos), no abstinentes y no consumidores, y b) asociaciones específicas entre alteraciones neuro-metabólicas del sistema de recompensa con rasgos de personalidad diferenciales para TUS y TUA abstinentes, no abstinentes y no consumidores.

\section{Método}

\section{Participantes}

Se seleccionaron 113 participantes divididos en abstinentes, no abstinentes y controles, con edades entre 18-45 años, reclutados en un servicio de salud mental ambulatoria, con diagnóstico de trastornos de personalidad (TP) (Esquizotípica, Antisocial, Borderline, Histriónico, Obsesiva-Compulsiva y Evitativa) (tabla 1).

Tabla 1

Datos socio-demográficos de los participantes

\begin{tabular}{|c|c|c|c|c|c|}
\hline & & No consumidores ( $N=32$ ) & Abstinentes $(\mathrm{N}=34)$ & No abstinentes ( $\mathrm{N}=37$ ) & $\mathrm{F}$ \\
\hline \multicolumn{2}{|c|}{ Edad años (media/DE) } & $32.08(12.6)$ & $34.31(11.8)$ & $39.12(14.1)$ & .35 \\
\hline \multirow{2}{*}{ Género } & Hombre & $17(53 \%)$ & $19(56 \%)$ & $20(54 \%)$ & .43 \\
\hline & Mujer & $15(47 \%)$ & $15(44 \%)$ & $17(46 \%)$ & .39 \\
\hline \multirow{2}{*}{ Estado civil } & Soltero & $14(43 \%)$ & $16(47 \%)$ & $18(49 \%)$ & .22 \\
\hline & Casado/separado & $18(57 \%)$ & 18 (53\%) & 19 (51\%) & .27 \\
\hline \multirow[t]{4}{*}{ Educación } & Primaria & 19 (59\%) & 20 (59\%) & $20(54 \%)$ & .14 \\
\hline & Secundaria/universitaria & $13(41 \%)$ & $14(41 \%)$ & $17(46 \%)$ & .11 \\
\hline & Empleado & $15(43.7 \%)$ & $17(50 \%)$ & $17(46 \%)$ & .35 \\
\hline & Estudiante & $3(9.4 \%))$ & $3(9 \%)$ & $5(13 \%)$ & .28 \\
\hline \multirow{2}{*}{ Ocupación } & \multirow[t]{2}{*}{ Desocupado } & $7(22 \%)$ & $5(14.7 \%)$ & $5(13 \%)$ & .17 \\
\hline & & $7(22 \%)$ & $9(26.3 \%)$ & $10(28 \%)$ & .19 \\
\hline \multirow[t]{2}{*}{ Sustancia d } & Alcohol & - & $16(47 \%)$ & $19(51 \%)$ & .12 \\
\hline & Otras sustancias & - & $18(53 \%)$ & $18(49 \%)$ & .09 \\
\hline \multicolumn{2}{|l|}{ Fumadores } & $17(53 \%)$ & $18(53 \%)$ & $24(65 \%)$ & .26 \\
\hline \multicolumn{2}{|c|}{ Inventario depresión de Beck } & $11.4(2.3)$ & $14.9(2.6)$ & $15.1(3.4)$ & .24 \\
\hline \multicolumn{2}{|c|}{ STAI-R (rango 0-100) } & $68.2(6.2)$ & $71.3(7.2)$ & $74.9(5.9)$ & .33 \\
\hline \multicolumn{2}{|c|}{ Trastorno psiquiátrico co-mórbido } & $9(28 \%)$ & $12(35 \%)$ & 13(37\%) & .10 \\
\hline \multicolumn{2}{|c|}{ Consumo de alcohol } & - & 21( & & \\
\hline \multicolumn{2}{|c|}{ Consumo de cocaina } & - & & & \\
\hline \multicolumn{2}{|c|}{ Severidad de adicción } & - & $6.5(1.3)$ & $7.1(1.1)$ & .21 \\
\hline \multicolumn{2}{|c|}{$\operatorname{IMC}\left(\mathrm{kg} / \mathrm{m}^{2}\right)^{*}$} & $25.2(2.1)$ & $26.2(3.1)$ & $24.6(3.9)$ & .12 \\
\hline
\end{tabular}

*IMC=indice de masa corporal 
Del total de 113 participantes, 32 eran no consumidores (grupo control), 71 tenían diagnóstico de Trastorno por uso de Alcohol o por uso de Sustancias, de los cuales 34 estaban abstinentes y 37 con consumo activo. Abstinentes y no abstinentes fueron equivalentes en consumo de alcohol y cocaína, severidad de adicción (medida con la EuropASI), co-morbilidad (depresión mayor o trastorno del estado de ánimo inducido por sustancias), trastornos médicos (hipertensión arterial y hepatitis $\mathrm{C}$ ), resultados del inventario de Beck y de ansiedad Estado-Rasgo, forma Y-2 ni en Índice de masa corporal que puede influir en los niveles de neurometabolitos (Gazdzinski, 2010). El 40\% tomaba antidepresivos (citalopram y escitalopram, dosis media $=15.30 \pm 3.8 \mathrm{mg} / \mathrm{dia}$ ), y $60 \%$ tomaba antihipertensivos (enalapril, dosis media $=12.4 \pm 4.3$; atenolol, $110 \pm 15.4 \mathrm{mg} /$ día; bisoprolol, $22.4 \pm 6.7 \mathrm{mg} /$ día). Los criterios de exclusión fueron historia de tumor cerebral, VIH/SIDA, accidente cerebrovascular, aneurisma cerebral, malformaciones arteriovenosas, enfermedad vascular periférica, infarto de miocardio, hipertensión arterial no controlada (presión sistólica>180 $\mathrm{mmHg}$ y/o diastólica>120 mmHg), diabetes Tipo 1, enfermedad pulmonar obstructiva crónica, convulsiones no relacionadas con consumo de sustancias, exposición a neurotoxinas (tolueno 0 tetracloruro de carbono), enfermedades degenerativas, síndrome de Wernicke-Korsakoff, demencia, traumatismo craneano con pérdida de conciencia > 10 minutos. Todos los participantes firmaron su consentimiento informado y se respetaron los protocolos del Comité Local de Ética. Los participantes fueron distribuidos en no consumidores, abstinentes y no abstinentes para Trastorno por uso de Sustancias/Trastorno por uso de Alcohol, basados en investigaciones que establecen diferencias entre estos dos últimos (Shedler y Block, 1990). Para el alcohol y otras sustancias se consideraron abstinentes quienes no habían consumido en el último año y no abstinentes quienes consumieron drogas o no menos de 6 tragos por semana en el mismo plazo. El control de la abstinencia se realizó mediante test toxicológico urinario de 6 Drogas (Cocaína, Anfetaminas, Metanfetaminas, Cannabis, Opiáceos, y Benzodiacepinas) con Prueba Rápida de Detección Instant-View (Alfa Scientific Designs, Inc, Cal) y detección de alcohol en aliento con alcoholímetro Alcosensor FST IV (Intoximeters, St. Louis, M0).

\section{Instrumentos}

Escala EuropASI (Bobes, 1996) es una entrevista semiestructurada y heteroaplicada que evalúa gravedad de la adicción (0-9), situación médica (16 ítems), situación laboral/soportes (26 items), consumo de alcohol y otras drogas (28 items), problemas legales (23 items), relaciones familiares y sociales (26 items) y estado psiquiátrico (22 items). Tiene consistencia interna de 0.62 en subescala de drogas, 0.87 en subescala de alcohol y enfermedades psiquiátricas y fiabilidad test-retest de 0.92 .

Inventario de depresión de Beck. El estado de ánimo depresivo se evaluó con el inventario de depresión de Beck (Beck, 1978).

Cuestionario de autoevaluación de Ansiedad-Rasgo (STAI-R) (Spielberger, 1982). Tiene consistencia interna entre .84 y .87 y valora la ansiedad-rasgo como predisposición a percibir situaciones como amenazantes; consta de 20 ítems con una puntuación de 0 y 60 y punto de corte de 25 para hombres y 32 para mujeres.
Escala de Impulsividad de Barratt (BIS-11) (Oquendo, 2001) consta de 30 preguntas auto-administradas con 3 subescalas: Impulsividad Cognitiva, Impulsividad Motora e Impulsividad no Planeada. La fiabilidad de la versión española es de .56.

Entrevista Clínica Estructurada para los Trastornos de Personalidad del Eje II (SCID), que utiliza un cuestionario y entrevistas semi-estructuradas (First, 1999). Tiene 119 ítems y 11 preguntas de respuestas abiertas y cerradas. Tiene fiabilidad inter-jueces $\mathrm{K}$ entre 0.24 y 0.74 y test-re-test de 0.37 a 0.84 .

Escala de Valoración Dimensional de las Patologías de la Personalidad-cuestionario básico (EI DAPP-BQ). Se empleó la adaptación española de Gutiérrez-Zotes (2008) con alta consistencia interna (a de Cronbach $=.93$ ) y coeficiente de correlación de .07 . Tiene 290 ítems y 5 opciones de respuesta, incluyendo 18 dimensiones que se agruparon en factores de segundo orden: emocional, desregulación, comportamiento disocial, inhibición y compulsividad.

\section{Procedimientos}

El diagnóstico de los trastornos por uso de alcohol y por uso de sustancias se realizó con entrevistas diagnósticas semi-estructuradas del manual de Entrevista Clínica Estructurada para los Trastornos del Eje I. No se estableció una diferencia entre abuso y dependencia (Sher, 2005) ya que forman un único continuo (Helzer, 2008), ni entre tipos de TUS para preservar la potencia estadística. Las evaluaciones espectroscópicas se realizaron con un resonador de 2T Unity/Inova (Varian NMR Instruments, Palo Alto, CA). El protocolo de Resonancia Magnética espectroscópica consistió en la adquisición anatómica y espectral de los datos, mediante segmentación tisular y localización de voxels en puntos de interés (CPFDL, CCA, ínsula, CRS y vermis cerebeloso) utilizando el método PRESS (point-resolved spectroscopic sequence) con adquisición rápida con secuencias de eco doble espin (TR/TE/TI=9.7/4/300 ms; resolución $1 \times 1 \mathrm{~mm}^{2}$; número de promedios $=128$, tiempo de adquisición $<5$ minutos). Las regiones de interés se derivaron de los datos suministrados en investigaciones previas asociadas con circuitos de toma de decisiones y descontrol de impulsos (Brewer y Potenza, 2008). El rastrillado manual de cada voxel produjo un ancho de línea de señal acuosa no suprimida menor a $11 \mathrm{~Hz}$. El procesamiento de datos espectroscópicos se realizó con paquete de ajuste espectral automático modelo LC (versión 6.2-1F) (Figura 1).

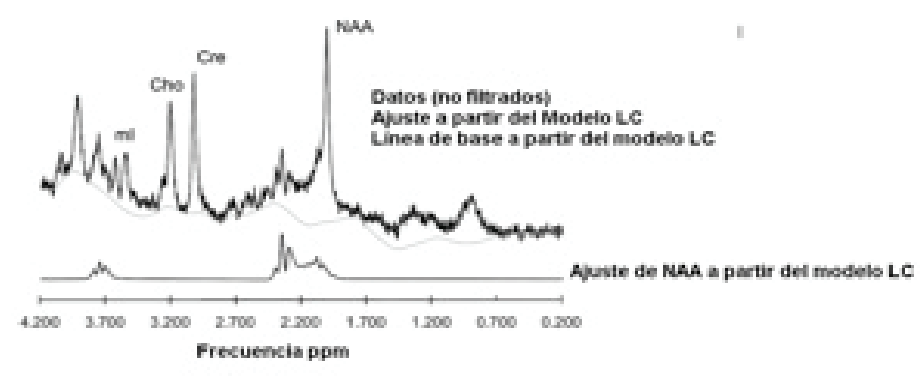

Figura 1. Resonancia magnética espectroscópica de corteza cingulada anterior ( $T E=25 \mathrm{~ms}$ ) con método PRESS junto con imagen espectral de NAA, Col, $\mathrm{Cr}$ y $\mathrm{ml}$. Se grafican los datos reales, el ajuste de datos a partir del modelo $\mathrm{LC}$, la línea de base y la línea de ajuste para NAA (Cho=Colina; $\mathrm{Cr}=$ Creatina; $\mathrm{NAA}=\mathrm{N}$-Acetil Aspartato; $\mathrm{ml}=$ mio-inositol; ppm=partes por millón). 
La volumetría regional y tisular T1-weighted de cerebro y cerebelo se realizó mediante segmentación probabilística automática (Gasparovic, 2006; Desikan, 2006; Thomson, 2012). Las concentraciones de $\mathrm{N}$-acetil aspartato (NAA), Colina (Co), creatina $(\mathrm{Cr})$ y mioinositol $(\mathrm{ml})$ se obtuvieron por análisis ${ }^{1} \mathrm{H}$ MRSI multicorte $(\mathrm{TR} / \mathrm{TI} / \mathrm{TE}=1.800 / 165 / 25 \mathrm{~ms})$. La relación NAA/Cr se obtuvo a partir de los radios de áreas de cada pico espectral. Los niveles de neurometabolitos se mostraron junto con la imagen anatómica con un programa SITOOLS (Brand, 1993), y las imágenes espectrales se procesaron con un programa LC (Soher, 1998) (figura 2)

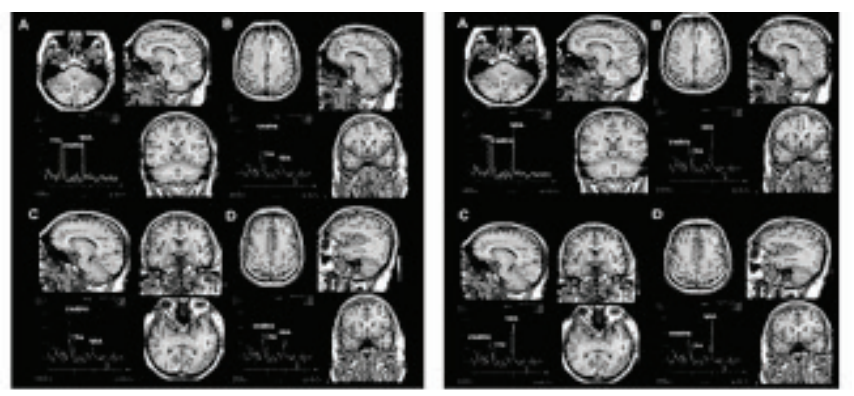

Figura 2. Ejemplos de resonancia magnética espectroscópica de abstinentes (izquierda) y no abstinentes (derecha) en las regiones de interés $(A=C P F D L, B=C C A, C=$ Ínsula y $D=$ vermis cerebeloso).

\section{Análisis de datos}

Se compararon los 3 grupos con chi cuadrado $(x 2)$ para variables categóricas, media de diferencias (test $t$ de Student de dos colas) y ANOVA con comparaciones múltiples lineales y curvilíneas para variables continuas. La normalidad de resultados del cuestionario se evaluó con el test de Kolmogorov-Smirnov y el DAPP-BO entre los grupos, con edad y género como co-variables se analizó con test de ANCOVA con corrección de Bonferroni. Se computó tamaño de efecto con $d$ de Cohen (1988) y omega cuadrada ( $\omega 2$ ) de grupos usando la fórmula de Hays (1981) para evitar sesgo por muestras. El DAPP-BO para TUA y TUS se evaluó con Signed Rank Test de Wilcoxon para muestras apareadas. Se colapsaron las categorías entre TUS y TUA independientemente del consumo pasado o presente, creando 4 grupos de TUS, TUA, no consumidores y consumidores con co-morbilidades a lo largo de la vida y se empleó ANOVA para detectar diferencias entre ellos. Los neurometablitos en no consumidores, abstinentes y no abstinentes, y en participantes con diferentes rasgos de personalidad patológica se compararon con análisis multivariado y tests $t$ apareado de dos colas medidos en CPFDL ( $r=.63)$, CCA $(r=.72)$, ínsula ( $r=.61)$, CRS ( $r=.61)$ y vermis cerebeloso $(r=.60)$. La tasa de significación $p$ se fijó en de .01 .

\section{Resultados}

La tabla 2 muestra las diferencias de rasgos para los sujetos no consumidores, abstinentes y no abstinentes para Trastorno por uso de Sustancias y Trastorno por uso de Alcohol.
Desregulación emocional $[t(31)=1.33, p<0.05]$, inhibición, $[t(31)=3.15, p<0.08]$ y expresión restringida, $[t(31)=3.53$, $p 0<.06$ ] distinguieron a consumidores (abstinentes y no abstinentes); y auto-agresión $[t(31)=1.75, p<0.01]$ comportamiento disocial, búsqueda de estímulos $[t(31)=3.61, p<0.01] y$ problemas de intimidad distinguieron a los no abstinentes. TUS y TUA se distinguieron entre si en desregulación emocional y búsqueda de estímulos. Los valores de impulsividad en los tres grupos se muestran en tabla 3.

Muchos rasgos desadaptativos, tales como compulsividad, problemas de conducta, evitación social, dureza fueron mayores en los no abstinentes que en los no consumidores.. Oposicionismo fue mayor en los participantes con Trastorno por uso de Sustancias no abstinentes con respecto a los abstinentes, $[t(31)=3.14, p<0.01]$ y no consumidores, $[t(31)=2.49, p<0.02]$. La tabla 4 muestra los resultados de la DAPP-BO para los sujetos no consumidores, consumidores TUA (abstinentes y no abstinentes), consumidores TUS (abstinentes y no abstinentes) y consumidores con co-morbilidades.

La desregulación emocional y compulsividad fueron mayores para Trastorno por uso de Sustancias y consumidores con co-morbilidades, incluyendo todas las sub-escalas excepto sumisión. La búsqueda de estímulos fue significativamente mayor en consumidores con co-morbilidades que en TUS y TUA, y estos últimos superaron a no consumidores. Mayor puntaje en narcisismo para TUS $\left(p<.001, \eta^{2}\right.$ parcial $\left.=.348\right)$ y en apego inseguro para TUA $\left(p<.002, \eta^{2}\right.$ parcial $\left.=.461\right)$ permitió diferenciarlos. La comparación entre abstinentes, no abstinentes y no consumidores de metabolitos cerebrales arrojó los siguientes resultados (tabla 5).

Corteza Prefrontal Dorsolateral: Se encontraron diferencias de grupo ( $\mathrm{p} \leq .003)$ para NAA $\left[\mathrm{X}^{2}(3)=17.43, \mathrm{p}<.001\right] \mathrm{Cr}$ $\left[X^{2}(3)=12.33, p=.004\right]$, pero no para Co o ml. Tests $t$ de Student de seguimiento ( $p$ corregida $\leq .023$ ) indicaron que no abstinentes exhibian menores niveles de NAA y $\mathrm{Cr}$ que abstinentes y no consumidores (ambas $p \leq .003$ ),

Corteza Cingulada Anterior: Hubo diferencias entre gru$\operatorname{pos}(p \leq .022)$ para NAA $\left[X^{2}(3)=11.47, p=.010\right]$ y Co $\left[X^{2}(3)=\right.$ $4.45, p=.057]$, pero no para $\mathrm{Cr}$ y ml. Tests $t$ de Student de seguimiento ( $p$ corregida $\leq .027$ ) mostraron que no abstinentes tuvieron menores niveles de NAA que no consumidores ( $p$ $=.012)$ y abstinentes $(p=.002)$. Insula: hubo diferencias de grupo $(p \leq .017)$ para NAA $\left[\mathrm{X}^{2}(3)=7.43, p<.016\right]$, pero no para $\mathrm{Co}, \mathrm{Cr}, 0 \mathrm{ml}$. Tests $t$ de Student de seguimiento ( $\mathrm{p}$ corregida $\leq .023)$ indicaron que los no abstinentes tenian menores niveles de NAA que no consumidores $(p=.008)$ y abstinentes $(p=.021)$.

Vermis Cerebeloso: hubo diferencias de grupo $(p \leq .017)$ para NAA $\left[X^{2}(3)=12.48, p<.003\right], \operatorname{Cr}\left[X^{2}(3)=10.44, p=\right.$ $.003]$ y Co $\left[\mathrm{X}^{2}(3)=11.27, p<.001\right]$ pero no para ml. Tests $t$ de Student ( $p$ corregida $\leq .033$ ) mostró que no abstinentes tenian menores niveles de NAA y $\mathrm{Cr}$ que abstinentes y no consumidores (ambas $p \leq .004$ ). Los abstinentes tuvieron mayores niveles de Co que no abstinentes $(p<0.003)$, y estos últimos mayores niveles respecto a no consumidores $(p<$ 0.007) 
Tabla 2

Resultados de la escala DAPP-BQ en los tres grupos de sujetos

\begin{tabular}{|c|c|c|c|c|c|c|c|}
\hline Dominio/dimensiones DAPP-BO $(\mathrm{N}=103)$ & $\begin{array}{c}\text { No consumidor } \\
\quad(n=32)\end{array}$ & $\begin{array}{l}\text { Abstinente } \\
(n=34)\end{array}$ & $\begin{array}{c}\text { No Abstinente } \\
(n=37)\end{array}$ & $\mathrm{F}^{*}$ & $w^{2}$ & $\beta_{\text {lineal }}$ & $\beta_{\text {curvilineal }}$ \\
\hline \multicolumn{8}{|l|}{ TUA } \\
\hline Auto-agresión $^{b}$ & 18.34 & 23.73 & 26.74 & 7.09 & 0.125 & .22 & -.14 \\
\hline Desregulación emocional $^{b c}$ & 249.32 & 365.33 & 438.23 & 24.08 & 0.833 & -.32 & .22 \\
\hline Labilidad afectiva $^{b c}$ & 31.31 & 30.87 & 45.69 & 12.98 & 0.324 & -.36 & .14 \\
\hline Ansiedad bc & 35.26 & 41.34 & 47.82 & 16.82 & 0.377 & -.42 & .16 \\
\hline Sumisión bc & 28.81 & 36.31 & 41.31 & 9.86 & 0.312 & -.41 & -.25 \\
\hline Apego inseguro ${ }^{b}$ & 37.47 & 40.76 & 52.41 & 9.53 & 0.223 & -.37 & -.48 \\
\hline Evitación social $^{\text {bc }}$ & 39.32 & 40.91 & 47.31 & 20.84 & 0.215 & -.26 & .09 \\
\hline alteración de la identidad ${ }^{b c}$ & 21.21 & 35.45 & 42.68 & 16.43 & 0.377 & -.01 & .22 \\
\hline Oposicionismo bc & 26.39 & 41.56 & 46.82 & 13.54 & 0.412 & -.29 & .31 \\
\hline Desregulación cognitiva ${ }^{a b}$ & 24.51 & 28.54 & 41.49 & 20.92 & 0.252 & -.18 & -.35 \\
\hline Narcisismo bc & 36.71 & 40.63 & 45.72 & 10.87 & 0.188 & -.08 & -.47 \\
\hline Suspicacia $^{\text {ab }}$ & 22.41 & 24.65 & 45.76 & 20.72 & 0.217 & -.28 & -.42 \\
\hline Comportamiento disocial $^{\mathrm{ab}}$ & 109.46 & 125.65 & 154.69 & 9.45 & 0.275 & -.31 & -.26 \\
\hline Problemas de conducta ${ }^{a b}$ & 21.41 & 23.45 & 34.61 & 5.08 & 0.312 & -.46 & -.52 \\
\hline Rechazo $^{\mathrm{ab}}$ & 24.68 & 28.65 & 37.12 & 6.97 & 0.453 & -.37 & -.32 \\
\hline Dureza $^{a b}$ & 24.81 & 28.23 & 38.34 & 8.64 & 0.326 & -.22 & .11 \\
\hline Búsqueda de estimulos ${ }^{b c}$ & 31.93 & 36.12 & 41.59 & 5.23 & 0.365 & .28 & .19 \\
\hline Inhibición bc & 70.81 & 76.34 & 80.26 & 12.96 & 0.275 & .05 & .32 \\
\hline Problemas de intimidad & 33.16 & 34.12 & 36.39 & 4.72 & 0.233 & .03 & .46 \\
\hline Expresión restringida ${ }^{b c}$ & 37.81 & 40.35 & 45.68 & 15.43 & 0.173 & -.26 & -.32 \\
\hline Compulsividad & 44.72 & 47.65 & 56.90 & 7.92 & 0.412 & -.33 & -.22 \\
\hline \multicolumn{8}{|l|}{ TAS } \\
\hline Auto-agresión ${ }^{\mathrm{ab}}$ & 21.31 & 24.89 & 27.84 & 8.19 & 0.115 & -.28 & .12 \\
\hline Desregulación emocional $^{a}$ & 326.61 & 379.33 & 441.28 & 25.18 & 0.354 & -.25 & -.25 \\
\hline Labilidad afectivabc & 32.59 & 34.17 & 47.19 & 15.54 & 0.239 & -.41 & -.42 \\
\hline Ansiedad ${ }^{b c}$ & 35.61 & 43.14 & 46.19 & 17.29 & 0.328 & -.32 & -.53 \\
\hline Sumisión bc & 31.49 & 38.41 & 43.51 & 9.70 & 0.395 & -.36 & -.32 \\
\hline Apego inseguro ${ }^{a b}$ & 36.71 & 42.49 & 53.72 & 9.70 & 0.269 & -.29 & -.36 \\
\hline Evitación social $^{\text {bc }}$ & 37.99 & 43.82 & 49.27 & 22.71 & 0.319 & -.41 & -.37 \\
\hline Alteración de la identidad ${ }^{b c}$ & 29.40 & 36.39 & 43.72 & 17.59 & 0.391 & -.19 & -.15 \\
\hline Oposicionismo $^{\text {abc }}$ & 38.22 & 44.67 & 47.91 & 14.67 & 0.409 & -.22 & -.17 \\
\hline Desregulación cognitiva ${ }^{a b}$ & 22.57 & 29.20 & 44.29 & 21.59 & 0.297 & -.29 & -.21 \\
\hline Narcisismo ${ }^{b}$ & 32.90 & 41.62 & 46.81 & 12.91 & 0.193 & -.31 & -.42 \\
\hline Suspicacia $^{b}$ & 20.98 & 24.71 & 47.41 & 22.92 & 0.206 & -.39 & -.27 \\
\hline Comportamiento disocial $^{a}$ & 112.94 & 131.49 & 159.49 & 10.34 & 0.270 & -.22 & -.44 \\
\hline Problemas de conducta ${ }^{a b}$ & 20.12 & 24.39 & 36.70 & 6.11 & 0.392 & .09 & -.45 \\
\hline Rechazo $^{b}$ & 22.87 & 29.65 & 38.32 & 7.90 & 0.423 & .22 & -.41 \\
\hline Dureza $^{\text {ab }}$ & 21.04 & 29.13 & 39.14 & 9.84 & 0.427 & .20 & -.23 \\
\hline Búsqueda de estimulos ${ }^{\mathrm{ab}}$ & 30.91 & 35.02 & 45.29 & 7.93 & 0.339 & -.04 & -.09 \\
\hline Inhibición bc & 71.50 & 77.94 & 84.16 & 14.06 & 0.225 & .15 & -.08 \\
\hline Problemas de intimidad ${ }^{a b}$ & 29.81 & 35.72 & 38.31 & 5.79 & 0.273 & .32 & -.27 \\
\hline Expresión restringida ${ }^{b c}$ & 32.08 & 43.15 & 45.88 & 17.23 & 0.163 & -.34 & -.26 \\
\hline Compulsividad $^{\mathrm{ab}}$ & 43.09 & 48.25 & 57.99 & 8.12 & 0.420 & -.21 & -.36 \\
\hline
\end{tabular}

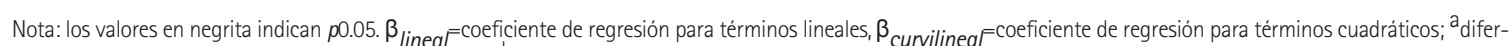
encia significativa entre no consumidores y abstinentes, ${ }^{b}$ diferencia significativa entre no consumidores y no abstinentes, ${ }^{c}$ diferencias significativas entre no abstinentes $y$ abstinentes. 
Tabla 3

Niveles de impulsividad en los 3 grupos de sujetos

\begin{tabular}{lllllll}
\hline Subescala & No consumidores & Abstinentes & No abstinentes & $\mathrm{P}$ & $\mathrm{w}^{2}$ & $\beta_{\text {lineal }}$ \\
\hline BIS 11 Totalbc & $51.3(4.5)$ & $64.3(6.8)$ & $66.9(7.8)$ & .007 & .134 & .07 \\
Impulsividad Cognitiva & $12.3(3.5)$ & $15.7(6.7)$ & $19.8(5.8)$ & .012 & .151 & -.09 \\
Impulsividad Motorabc & $17.3(4.7)$ & $21.8(7.8)$ & $25.9(3.9)$ & .006 & .138 & -.13 \\
Impulsividad No planeadabc & $17.9(3.9)$ & $20.7(3.9)$ & $22.7(5.2)$ & .007 & .129 & -.08 \\
\hline
\end{tabular}

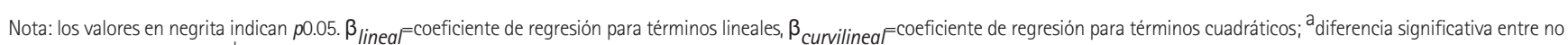
consumidores y abstinentes; ${ }^{b}$ diferencia significativa entre no consumidores y no abstinentes, ${ }^{c}$ diferencias significativas entre no abstinentes y abstinentes.

Tabla 4

Análisis de rasgos de personalidad para sujetos no consumidores y consumidores TUS, TUA, y con co-morbilidad a lo largo de la vida

\begin{tabular}{|c|c|c|c|c|c|c|c|}
\hline Rasgo & $\begin{array}{l}\text { No consumidores } \\
(\mathrm{N}=32)\end{array}$ & $\begin{array}{l}\text { Consumidores TUA } \\
(\mathrm{N}=35)\end{array}$ & $\begin{array}{l}\text { Consumidores TUS } \\
(\mathrm{N}=36)\end{array}$ & $\begin{array}{l}\text { Consumidores } \\
\text { c/ comorbilidad } \\
(\mathrm{N}=25)\end{array}$ & $\mathrm{F}$ & $\beta_{\text {lineal }}$ & $\beta_{\text {curvilineal }}$ \\
\hline Auto-agresión abd & 23.41 & 25.76 & 28.32 & 28.29 & $7.14^{*}$ & .05 & -.12 \\
\hline Desregulación emocional ${ }^{\text {abcd }}$ & 324.52 & 380.24 & 442.19 & 396.09 & $6.35^{*}$ & .11 & .21 \\
\hline Labilidad afectiva $^{\text {bcd }}$ & 33.48 & 35.08 & 48.00 & 48.46 & $7.14^{*}$ & .03 & -.23 \\
\hline Ansiedad $^{\text {bc }}$ & 36.52 & 44.05 & 47.01 & 47.10 & $6.23^{*}$ & .11 & -.32 \\
\hline Sumisión ${ }^{\text {abd }}$ & 32.20 & 39.32 & 40.42 & 40.61 & 4.40 & -.09 & .09 \\
\hline Apego inseguro bc & 36.62 & 45.30 & 40.63 & 41.61 & 7.38 & -.12 & -.08 \\
\hline Evitación social $^{b c d}$ & 36.80 & 49.73 & 47.32 & 48.62 & 7.25 & -.23 & -.16 \\
\hline Alteración de la identidad ${ }^{\text {acd }}$ & 29.37 & 36.35 & 43.63 & 47.42 & 7.45 & -.31 & -.25 \\
\hline Oposicionismo bc & 38.30 & 44.58 & 47.83 & 48.69 & $7.41^{*}$ & .09 & -.31 \\
\hline Desregulación cognitiva & 22.49 & 29.18 & 44.19 & 41.47 & 8.29 & .12 & -.43 \\
\hline Narcisismo ${ }^{\text {bcd }}$ & 32.61 & 41.54 & 46.72 & 47.05 & $8.59^{*}$ & .17 & .09 \\
\hline Suspicacia & 20.67 & 24.62 & 47.32 & 42.84 & 6.20 & -.12 & .11 \\
\hline Comportamiento disocial $^{\text {bcd }}$ & 112.86 & 141.39 & 149.34 & 150.42 & $6.17^{*}$ & .21 & .18 \\
\hline Problemas de conducta $^{b c}$ & 20.04 & 34.29 & 36.61 & 46.61 & $8.34^{*}$ & .31 & .21 \\
\hline Rechazo & 22.79 & 39.58 & 38.23 & 47.47 & 7.42 & .24 & -.14 \\
\hline Dureza & 26.18 & 39.93 & 39.05 & 49.12 & 8.74 & -.36 & -.37 \\
\hline Búsqueda de estímulos ${ }^{\text {bcd }}$ & 34.82 & 37.82 & 45.10 & 47.53 & $7.41^{* *}$ & -.31 & -.36 \\
\hline Inhibición bcd & 71.41 & 77.85 & 84.07 & 84.16 & $9.28^{*}$ & -.21 & -.21 \\
\hline Problemas de intimidad & 29.72 & 35.64 & 38.23 & 45.59 & 6.26 & .16 & .09 \\
\hline Expresión restringida & 34.99 & 43.06 & 45.77 & 57.63 & 7.19 & .16 & .18 \\
\hline Compulsividad $^{\text {bcd }}$ & 43.09 & 48.16 & 57.76 & 58.37 & $8.40^{* *}$ & .21 & .32 \\
\hline
\end{tabular}

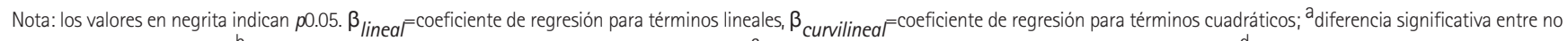
consumidores y abstinentes; ${ }^{b}$ diferencia significativa entre no consumidores y no abstinentes, ${ }^{c}$ diferencias significativas entre no abstinentes y abstinentes, ${ }^{d}$ diferencias significativas entre abstinentes $y$ co-morbilidad 
Tabla 5

Resultados de metabolitos cerebrales en los tres grupos de participantes

\begin{tabular}{|c|c|c|c|c|c|c|}
\hline \multirow[b]{2}{*}{ Región } & \multirow[b]{2}{*}{ Metabolito } & \multirow[b]{2}{*}{ No consumidores } & \multirow[b]{2}{*}{ Abstinentes } & \multirow[b]{2}{*}{ No abstinentes } & \multicolumn{2}{|c|}{ Tamaño de efecto (d de Cohen) } \\
\hline & & & & & $\begin{array}{l}\text { No abstinentes vs. } \\
\text { no consumidores }\end{array}$ & $\begin{array}{l}\text { No abstinentes vs. } \\
\text { Abstinentes }\end{array}$ \\
\hline \multirow{4}{*}{ CPFDL } & $\mathrm{NAA}(\mathrm{mM} / \mathrm{Kg}$ peso & $34.5(3.5)^{\mathrm{a}}$ & $36.3(4.5)^{b}$ & $31.1(3.5)^{\mathrm{ab}}$ & \multirow[t]{4}{*}{0.89} & \multirow{4}{*}{$\begin{array}{l}1.10 \\
0.98\end{array}$} \\
\hline & $\begin{array}{l}\mathrm{Cr}(\mathrm{mM} / \mathrm{Kg} \text { peso } \\
\text { húmedo) }\end{array}$ & $22.3(2.3)$ & $23.9(2.4)^{b}$ & $21.7(2.6)^{b}$ & & \\
\hline & $\begin{array}{l}\text { Col(mM/Kg peso } \\
\text { húmedo) }\end{array}$ & $5.2(0.9)$ & $5.7(0.9)$ & $5.1(0.8)$ & & \\
\hline & $\begin{array}{l}\mathrm{ml}(\mathrm{mM} / \mathrm{Kg} \text { peso } \\
\text { húmedo) }\end{array}$ & $17.4(3.4)$ & $19.7(4.5)$ & $18.4(3.3)$ & & \\
\hline \multirow{4}{*}{ CCA } & $\begin{array}{l}\text { NAA(mM/Kg peso } \\
\text { húmedo) }\end{array}$ & $312(3.6)^{a}$ & $31.3(3.5)^{b}$ & $25.1(4.6)^{\mathrm{ab}}$ & \multirow[t]{4}{*}{0.65} & \multirow[t]{4}{*}{0.71} \\
\hline & $\begin{array}{l}\mathrm{Cr}(\mathrm{mM} / \mathrm{Kg} \text { peso } \\
\text { húmedo) }\end{array}$ & $20.3(1.3)$ & $21.4(3.4)$ & $20.6(3.7)$ & & \\
\hline & $\begin{array}{l}\text { Col(mM/Kg peso } \\
\text { húmedo) }\end{array}$ & $6.2(0.9)$ & $6.6(0.9)$ & $6.0(0.8)$ & & \\
\hline & $\begin{array}{l}\mathrm{ml}(\mathrm{mM} / \mathrm{Kg} \text { peso } \\
\text { húmedo) }\end{array}$ & $18.4(4.4)$ & $19.9(3.5)$ & 18.1(3.4) & & \\
\hline \multirow{4}{*}{ Insula } & $\begin{array}{l}\text { NAA(mM/Kg peso } \\
\text { húmedo) }\end{array}$ & $33.5(2.5)^{\mathrm{a}}$ & $32.6(3.5)^{b}$ & $31.1(4.5)^{\mathrm{ab}}$ & \multirow[t]{4}{*}{0.68} & \multirow[t]{4}{*}{0.52} \\
\hline & $\begin{array}{l}\mathrm{Cr}(\mathrm{mM} / \mathrm{Kg} \text { peso } \\
\text { húmedo) }\end{array}$ & $23.3(3.3)$ & $23.2(2.9)$ & $22.7(3.6)$ & & \\
\hline & $\begin{array}{l}\text { Col(mM/Kg peso } \\
\text { húmedo) }\end{array}$ & $6.5(0.9)$ & $6.1(0.9)$ & $6.2(0.8)$ & & \\
\hline & $\begin{array}{l}\mathrm{ml}(\mathrm{mM} / \mathrm{Kg} \text { peso } \\
\text { húmedo) }\end{array}$ & $19.4(4.4)$ & $20.7(3.5)$ & $20.4(4.3)$ & & \\
\hline \multirow{4}{*}{ Vermis } & $\begin{array}{l}\text { NAA(mM/Kg peso } \\
\text { húmedo) }\end{array}$ & $33.5(3.7)^{a}$ & $35.3(5.5)^{b}$ & $31.4(5.5)^{\mathrm{ab}}$ & \multirow[t]{2}{*}{0.60} & \multirow[t]{2}{*}{$\begin{array}{l}1.10 \\
0.65\end{array}$} \\
\hline & $\begin{array}{l}\mathrm{Cr}(\mathrm{mM} / \mathrm{Kg} \text { peso } \\
\text { húmedo) }\end{array}$ & $32.3(1.3)$ & $33.9(5.4)^{b}$ & $31.7(4.6)^{b}$ & & \\
\hline & $\begin{array}{l}\text { Col(mM/Kg peso } \\
\text { húmedo) }\end{array}$ & $7.2(0.5)$ & $9.7(1.4)$ & $8.6(1.1)$ & \multirow[t]{2}{*}{0.79} & \multirow[t]{2}{*}{0.76} \\
\hline & $\begin{array}{l}\mathrm{ml}(\mathrm{mM} / \mathrm{Kg} \text { peso } \\
\text { húmedo) }\end{array}$ & $24.4(3.8)$ & $26.7(4.7)$ & $23.6(4.3)$ & & \\
\hline \multirow{4}{*}{ CRS } & $\begin{array}{l}\text { NAA(mM/Kg peso } \\
\text { húmedo) }\end{array}$ & $31.5(3.7)^{\mathrm{a}}$ & $31.3(4.6)^{b}$ & $28.3(4.5)^{\mathrm{ab}}$ & 0.76 & \multirow{4}{*}{$\begin{array}{l}0.72 \\
0.71 \\
0.74\end{array}$} \\
\hline & $\begin{array}{l}\mathrm{Cr}(\mathrm{mM} / \mathrm{Kg} \text { peso } \\
\text { húmedo) }\end{array}$ & 18.3(3.3) & $19.9(4.4)^{b}$ & $18.4(3.6)^{\mathrm{ab}}$ & \multirow[t]{3}{*}{0.86} & \\
\hline & $\begin{array}{l}\text { Col(mM/Kg peso } \\
\text { húmedo) }\end{array}$ & $5.9(0.9)$ & $5.8(0.9)^{b}$ & $5.3(0.8)^{a b}$ & & \\
\hline & $\begin{array}{l}\mathrm{ml}(\mathrm{mM} / \mathrm{Kg} \text { peso } \\
\text { húmedo) }\end{array}$ & $16.9(2.4)$ & $18.7(4.7)$ & $17.2(4.3)$ & & \\
\hline
\end{tabular}

Nota: $\mathrm{CPFDL}=$ corteza prefrontal dorsolateral; $\mathrm{CRS}=$ corona radiada superior; $\mathrm{CCA}=$ corteza cingulada anterior; $\mathrm{NAA}=\mathrm{N}$-acetil-aspartato; $\mathrm{Cr}=\mathrm{Creatina}$; $\mathrm{Col}=\mathrm{Colina}$; $\mathrm{ml}=\mathrm{mioinositol} \mathrm{a}_{\mathrm{no}}$ abstinente $<$ no consumidor, $p<.05 ;{ }^{b}$ no abstinente<abstinente, $p<.05$. 
Tabla 6

Correlación de Pearson entre rasgos de personalidad, metabolitos y TUA/TUS

\begin{tabular}{|c|c|c|c|c|c|c|c|c|c|c|}
\hline & & \multicolumn{5}{|c|}{ Rasgos Personalidad } & & \multirow{2}{*}{\multicolumn{3}{|c|}{ Metabolitos cerebrales }} \\
\hline & \multirow[b]{2}{*}{ a } & \multicolumn{4}{|c|}{ DAPP-BQ } & \multicolumn{2}{|c|}{ BIS 11} & & & \\
\hline & & B & c & $d$ & $\mathrm{E}$ & $f$ & g & h & i & j \\
\hline TUA/TUS no abstinentes & -.45 & -.43 & -.53 & -.57 & -.42 & -.59 & -.44 & -.47 & .51 & .63 \\
\hline a.TUA/TUS abstinentes & 1 & .68 & .65 & .32 & .43 & .51 & .46 & .39 & .36 & .41 \\
\hline b.Desregulación emotiva & & 1 & .65 & .57 & -.72 & -.47 & -.59 & -.62 & .44 & -36 \\
\hline c.Comportamiento disocial & & & 1 & -.72 & -.69 & -.66 & -.58 & -.49 & -.38 & -.28 \\
\hline d.Inhibición & & & & 1 & .71 & -.48 & .46 & .62 & .54 & .26 \\
\hline e.Compulsividad & & & & & 1 & .74 & -.47 & -.62 & .49 & -.38 \\
\hline f.BIS 11 & & & & & & 1 & .55 & .47 & .52 & .35 \\
\hline g.NAA/Cr CPFDL & & & & & & & 1 & -.69 & .63 & .49 \\
\hline h.NAA/Cr CCA & & & & & & & & 1 & .61 & -.48 \\
\hline I NAA/Cr Insula & & & & & & & & & 1 & -.30 \\
\hline $\mathrm{J} \mathrm{Co} / \mathrm{Cr}$ Vermis cerebeloso & & & & & & & & & & 1 \\
\hline
\end{tabular}

TUA/TUS no abstinentes= Trastornos por uso de alcohol/trastorno por uso de sustancias No Abstinentes prevalencia de por vida, TUA/TUS abstinentes=trastornos por uso de alcohol/trastorno por uso de sustancias Abstinentes prevalencia de por vida, DAPP-BQ=valoración dimensional trastornos de la personalidad-escala básica, BIS 11=escala de impusividad de Barrett, NAA/Cr CPFDL=relación N-acetil-aspartato/creatinina en corteza pre-frontal dorsolateral, NAA/Cr CCA=relación N-acetil-aspartato/creatinina en corteza cingulada anterior (valor normal 2.0). Todas las relaciones fueron significativas para $p<.001$.

Corona Radiada Superior: Hubo diferencias de grupo $(p \leq$ .010) en $\mathrm{NAA}_{1} \mathrm{X}^{2}(3)=15.63, p=.002 ; \mathrm{Cr}_{1} \mathrm{X}^{2}(3)=15.13, p=.002$; y Co, $X^{2}(3)=16.15, p=.002$; pero no para ml. Tests $t$ de Student $(p \leq .024)$ señalaron que no abstinentes tenían menores niveles de NAA, Cr y Co que no consumidores (todas las $p \leq .014$ ) y abstinentes $(p \leq .017)$. Hubo mayor nivel de Co en vermis cerebeloso para abstinentes. Las diferencias permanecieron significativas después de corregir para consumo de cigarrillos, co-morbilidades, ansiedad, depresión, género, educación o tipo de empleo. La correlación entre las variables utilizadas se muestra en tabla 6 .

La comparación entre trastorno por uso de sustancias a lo largo de la vida, trastorno por uso de alcohol a lo largo de la vida, consumidores con co-morbilidades y no consumidores frente a los niveles de neurometabolitos se expresa en la tabla 7.

La interacción entre sub-escalas de impulsividad de BIS-11 y neurometabolitos para las regiones de interés CPFDL, CCA, Insula, Corona radiada y Vermis Cerebeloso mostró un efecto general de grupo significativo $(F(3,102)=2.30, p<.022)$. A nivel individual se observó un efecto significativo entre Índice $\mathrm{Co} / \mathrm{Cr}$ e impulsividad no planeada en vermis cerebeloso $\left(p=.005, \eta^{2}\right.$ parcial $=.003$ ) y entre Índice $N A A / C r$ e impulsividad no planeada y motora en CPFDL ( $p=.015, \eta^{2}$ parcial $\left.=.026\right)$, Ínsula $(p=.05$, $\eta^{2}$ parcial $\left.=.014\right)$ y CCA $\left(p=.041, \eta^{2}\right.$ parcial $\left.=.017\right)$ (figura 3$)$.

\section{Discusión}

De la investigación surge evidencia de una relación entre rasgos de personalidad y TUS/TUA a diferencia de estudios previos (Ruiz, 2008). Los rasgos de personalidad se agruparon en tres patrones diferentes según rasgos dimensionales (abstinente y no abstinente) y estructurales (TUS/TUA y no consumidores). El primero presentó labilidad, autoagresión, narcisismo, apego inseguro y ansiedad asociados a no consumidores y abstinentes, aparentando ser un marcador de rasgo (Hopwood, 2007). El segundo grupo mostró suspicacia, rechazo y dureza en no abstinentes y abstinentes, exceptuando no consumidores, presentándose como un factor de estado. El tercer grupo ofreció compulsión, impulsividad (escalas corporal y no planeada) y desregulación emocional junto con bajos niveles de NAA y $\mathrm{Cr}$ en no abstinentes y participantes con co-morbilidad. Desapego e inhibición se asociaron con TUA y los rasgos narcisistas con TUS. De acuerdo con la primera hipótesis, adictos no abstinentes y con co-morbilidades se asociaron significativamente con rasgos de personalidad desadaptativos y desinhibitorios. De acuerdo con la segunda hipótesis, se encontró una asociación robusta entre rasgos de impulsividad, búsqueda de estímulos, compulsividad y menores niveles de NAA en CPFDL, CCA e ínsula en no abstinentes y con co-morbilidades.

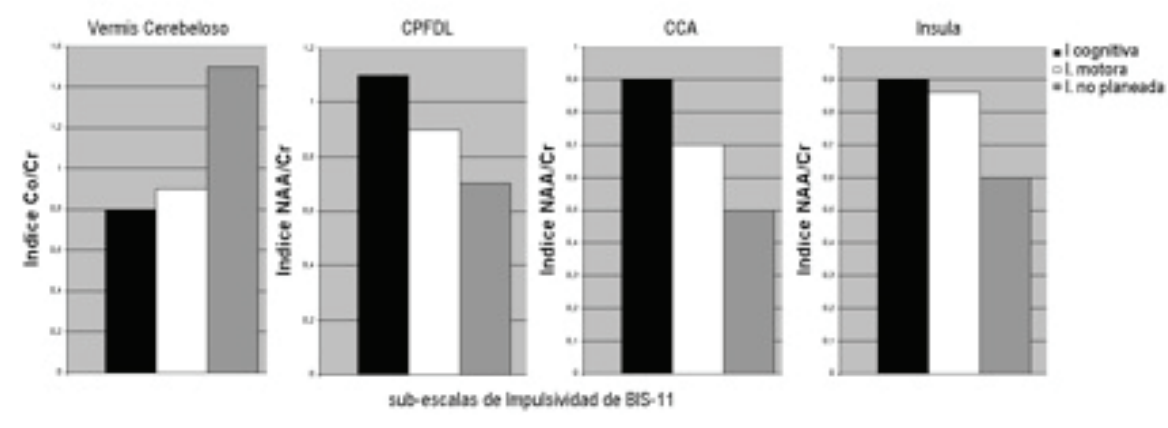

Figura 3. Interacción de metabolitos cerebrales y sub-escalas de BIS-11 
Tabla 7

Metabolitos cerebrales con relación a las categorías agrupadas de participantes

\begin{tabular}{|c|c|c|c|c|c|c|c|}
\hline \multirow[b]{2}{*}{ Región } & \multirow[b]{2}{*}{ Metabolito } & \multirow[b]{2}{*}{ No consumidores } & \multirow[b]{2}{*}{ TUA } & \multirow[b]{2}{*}{ TUS } & \multirow[b]{2}{*}{$\begin{array}{l}\text { Consumidores con } \\
\text { co-morbilidad }\end{array}$} & \multicolumn{2}{|c|}{ Tamaño de efecto (d de Cohen) } \\
\hline & & & & & & $\begin{array}{l}\text { No abstinentes vs. } \\
\text { no consumidores }\end{array}$ & $\begin{array}{l}\text { No abstinentes vs. } \\
\text { Abstinentes }\end{array}$ \\
\hline \multirow{4}{*}{ CPFDL } & $\begin{array}{l}\text { NAA (mM/Kg peso } \\
\text { húmedo) }\end{array}$ & $30.1(2.6)^{\mathrm{a}}$ & $27.2(5.4)^{b}$ & $40.2(4.6)^{\mathrm{ab}}$ & $22.0(4.4)^{a b}$ & \multirow[t]{4}{*}{0.70} & \multirow[t]{4}{*}{$\begin{array}{l}2.01 \\
1.87\end{array}$} \\
\hline & $\begin{array}{l}\mathrm{Cr}(\mathrm{mM} / \mathrm{Kg} \text { peso } \\
\text { húmedo) }\end{array}$ & $21.4(1.4)$ & $22.0(1.5)^{b}$ & $22.5(3.5)^{b}$ & $22.6(3.7)^{b}$ & & \\
\hline & $\begin{array}{l}\text { Col(mM/Kg peso } \\
\text { húmedo) }\end{array}$ & $4.3(1.8)$ & $5.7(0.9)$ & $5.1(0.8)$ & $5.1(0.8)$ & & \\
\hline & $\begin{array}{l}\mathrm{ml}(\mathrm{mM} / \mathrm{Kg} \text { peso } \\
\text { húmedo) }\end{array}$ & $16.3(2.5)$ & $16.7(4.5)$ & $17.4(2.6)$ & $17.3(2.8)$ & & \\
\hline \multirow{4}{*}{ CCA } & $\begin{array}{l}\text { NAA(mM/Kg peso } \\
\text { húmedo) }\end{array}$ & $32.1(4.5)^{\mathrm{a}}$ & $30.8(4.4)^{b}$ & $28.1(4.6)^{a}$ & $25.9(5.1)^{\mathrm{a}}$ & \multirow[t]{4}{*}{0.59} & \multirow[t]{4}{*}{0.68} \\
\hline & $\begin{array}{l}\mathrm{Cr}(\mathrm{mM} / \mathrm{Kg} \text { peso } \\
\text { húmedo) }\end{array}$ & $21.2(1.4)$ & $20.5(2.5)$ & $21.5(4.6)$ & $21.5(2.8)$ & & \\
\hline & $\begin{array}{l}\mathrm{Col}(\mathrm{mM} / \mathrm{Kg} \text { peso } \\
\text { húmedo) }\end{array}$ & $5.4(1.6)$ & $5.9(1.8)$ & $5.2(1.9)$ & $5.9(1.6)$ & & \\
\hline & $\begin{array}{l}\text { ml(mM/Kg peso } \\
\text { húmedo) }\end{array}$ & $17.3(3.6)$ & $18.3(2.5)$ & $17.2(4.1)$ & $16.3(2.9)$ & & \\
\hline \multirow{4}{*}{ Insula } & $\begin{array}{l}\text { NAA(mM/Kg peso } \\
\text { húmedo) }\end{array}$ & $34.2(1.7)^{\mathrm{a}}$ & $31.7(4.2)^{b}$ & $31.1(4.5)^{b}$ & $30.0(7.2)^{b}$ & \multirow[t]{4}{*}{0.77} & \multirow[t]{4}{*}{0.42} \\
\hline & $\begin{array}{l}\mathrm{Cr}(\mathrm{mM} / \mathrm{Kg} \text { peso } \\
\text { húmedo) }\end{array}$ & $22.4(2.5)$ & $24.1(3.3)$ & 22.4(3.6) & $23.5(3.6)$ & & \\
\hline & $\begin{array}{l}\text { Col(mM/Kg peso } \\
\text { húmedo) }\end{array}$ & $6.4(1.5)$ & $5.62(1.5)$ & $6.2(2.8)$ & $6.4(4.6)$ & & \\
\hline & $\begin{array}{l}\mathrm{ml}(\mathrm{mM} / \mathrm{Kg} \text { peso } \\
\text { húmedo) }\end{array}$ & $21.1(3.6)$ & 21.2(4.3) & $21.2(4.3)$ & $20.4(4.3)$ & & \\
\hline \multirow{4}{*}{ Vermis } & $\begin{array}{l}\text { NAA(mM/Kg peso } \\
\text { húmedo) }\end{array}$ & $32.6(2.9)^{a}$ & $34.6(4.9)^{b}$ & $30.5(5.5)^{\mathrm{ab}}$ & $33.6(4.9)^{b}$ & \multirow[t]{2}{*}{0.60} & \multirow[t]{2}{*}{$\begin{array}{l}1.15 \\
0.58\end{array}$} \\
\hline & $\begin{array}{l}\mathrm{Cr}(\mathrm{mM} / \mathrm{Kg} \text { peso } \\
\text { húmedo) }\end{array}$ & $31.4(2.1)$ & $31.0(4.6)^{b}$ & $30.4(9.6)^{b}$ & $31.7(5.6)^{b}$ & & \\
\hline & $\begin{array}{l}\text { Col(mM/Kg peso } \\
\text { húmedo) }\end{array}$ & $6.3(0.9)$ & $7.9(0.8)$ & $9.3(1.6)$ & $8.5(4.1)$ & \multirow[t]{2}{*}{0.82} & \multirow[t]{2}{*}{0.65} \\
\hline & $\begin{array}{l}\mathrm{ml}(\mathrm{mM} / \mathrm{Kg} \text { peso } \\
\text { húmedo) }\end{array}$ & $23.5(2.9)$ & $27.3(5.1)$ & $23.6(4.3)$ & $22.6(4.3)$ & & \\
\hline \multirow{4}{*}{ CRS } & $\begin{array}{l}\text { NAA(mM/Kg peso } \\
\text { húmedo) }\end{array}$ & $30.6(3.8)^{\mathrm{a}}$ & $30.5(3.7)^{6}$ & $28.3(4.5)^{\mathrm{a}}$ & $26.1(4.5)^{\mathrm{ab}}$ & \multirow[t]{4}{*}{0.59} & \multirow{4}{*}{0.53} \\
\hline & $\begin{array}{l}\operatorname{Cr}(\mathrm{mM} / \mathrm{Kg} \text { peso } \\
\text { húmedo) }\end{array}$ & $17.9(2.8)$ & $18.0(3.6)^{b}$ & $18.4(3.6)^{b}$ & $17.1(3.6)^{\mathrm{ab}}$ & & \\
\hline & $\begin{array}{l}\text { Col(mM/Kg peso } \\
\text { húmedo) }\end{array}$ & $5.6(1.8)$ & $5.9(1.3)^{b}$ & $5.1(1.5)$ & $5.7(1.3)^{\mathrm{ab}}$ & & \\
\hline & $\begin{array}{l}\text { ml(mM/Kg peso } \\
\text { húmedo) }\end{array}$ & $17.4(4.1)$ & $18.7(4.7)$ & $16.1(2.5)$ & $16.7(2.9)$ & & \\
\hline
\end{tabular}

Este descenso se atribuye a pérdida de neuronas o reducción de integridad neuronal, ya que este neurometabolito es intraneuronal (Meyeroff, 2013). Dado que dichas zonas cerebrales se asocian con toma de decisiones, resolución de problemas, control de impulsos y regulación afectiva (Verdejo-García, 2006) desempeñan un papel en la incapacidad para evitar el consumo reiterado, ansiedad y depresión. En cambio, menores niveles de NAA en vermis cerebeloso se relacionaron con abstinentes y no abstinentes, exceptuando los no consumidores (Paul, 2009), comprometiendo el aprendizaje de rutinas de evitación del consumo, dado que el cerebelo interviene en memoria de trabajo y aprendizajes implícitos asociados al procesamiento de la recompensa (Schulte, 2012) a través de conexiones recíprocas entre los lóbulos frontal, parietal, temporal, estructuras subcorticales (Mega y Cummings, 1994) y la corona radiada. Esta alteración representa un punto vulnerable en el ciclo de abstinencia-recaídas propios de las adicciones (Salazar-Fraile, 2010; Durazzo, 2011; Joos, 2013), aunque no se pudo determinar si es causa o efecto del consumo dada la naturaleza transversal del estudio. El mayor índice de $\mathrm{Co} / \mathrm{Cr}$ en vermis cerebeloso, que distingue a los abstinentes de alcohol, señala un incremento de la reparación de membranas axona- 
les o dendríticas en estos últimos. Estos resultados igualan los obtenidos en modelos animales de alteraciones metabólicas inducidas por alcohol (Tesner y Hill, 2010; Meyerhoff, 2013). Limitaciones: a) el diseño transversal no permite determinar si los rasgos patológicos representan una predisposición o los efectos del uso crónico en participantes en remisión y no abstinentes; b) la muestra pequeña podría reducir la potencia estadistica para evitar efectos nulos, y para investigar relaciones diferenciales entre rasgos patológicos y los distintos tipos de sustancias de adicción; c) podrían existir relaciones no lineales entre rasgos patológicos y TUS/TUA que no se pudieron identificar; d) tampoco se investigó la influencia del apoyo social o habilidades de afrontamiento sobre el consumo y recaídas (Walter, 2006).

En definitiva, los Trastornos por uso de Sustancias y Trastornos por uso de Alcohol, particularmente en no abstinentes se asocian con rasgos de personalidad desadaptativos y desinhibitorios, siendo más consistentes en los casos de co-morbilidad. Estos patrones se asocian con disminución de NAA en corteza prefrontal dorsolateral, corteza cingulada anterior y vermis cerebeloso, además de aumento del índice $\mathrm{Co} / \mathrm{Cr}$ en vermis cerebeloso. Estas modificaciones pueden desempeñar un papel en el mantenimiento o agravamiento del consumo o la recaída en ambos trastornos, mediados conjuntamente por alteraciones neurobiológicas y de personalidad.

\section{Referencias}

Ball, S. A. (2005). Personality traits, problems, and disorders: Clinical applications to substance use disorders. Journal of Research in Personality, 39, 84-102.

Ball, S. A., Carroll, K. M., Canning-Ball, M., y Rounsaville, B. J. (2006). Reasons for dropout from drug abuse treatment: Symptoms, personality, and motivation. Addictive Behaviors, 31, 320-330.

Bartha, R., Stein, M. B., Williamson, P. C., Drost, D. J., Neufeld, R. W. y Carr, T. J. (1998). A short echo $1 \mathrm{H}$ spectroscopy and volumetric MRI study of the corpus striatum in patients with obsessive-compulsive disorder and comparison subjects. American Journal of Psychiatry, 155: 1584-91

Beck, A. T. (1978). Depression Inventory. Philadelphia: PA, Center for Cognitive Therapy.

Bertolino, A., Esposito, G. y Callicott, J. H. (2000). Specific relationship between prefrontal neuronal $\mathrm{N}$-acetylaspartate and activation of the working memory cortical network in schizophrenia. American Journal of Psychiatry, 157, 26-33

Bobes, J., González, M. P., Sáiz, P. A. y Bousoño, M. (1996). Índice europeo de severidad de la adicción: EuropASI. Versión española, (pp. 201218), Actas de la IV Reunión Interregional de Psiquiatría,

Brand, A., Richter-Landsberg, C. y Leibfritz, D. (1993). Multinuclear NMR studies on the energy metabolism of glial and neuronal cells. Developmental Neuroscience, 15, 289-298.

Brewer, J. y Potenza, M. (2008). The Neurobiology and Genetics of Impulse control disorders. Relationships to drug addictions. Biochemical Pharmacology, 75, 63-75
Buckley, P. F. y Friedman, L. (2000). Magnetic resonance spectroscopy: Bridging the neurochemistry and neuroanatomy of schizophrenia. British Journal of Psychiatry, 176, 203-205

Cecil, K., DelBello, M., Sellars, M. C. y Strakowski, S. M. (2003). Proton magnetic resonance spectroscopy of the frontal lobe and cerebellar vermis in children with a mood disorder and a familial risk for bipolar disorders. Journal of Child \&t Adolescent Psychopharmacology, 13: 545-555

Cohen J. (1988). Statistical power analysis of the behavioral sciences. New York: Academic Press.

Davanzo, P., Yue, K., Thomas, M. A., Belin, T., Mintz, J. y Venkatraman, T. N. (2003). Proton magnetic resonance spectroscopy of bipolar disorder versus intermittent explosive disorder in children and adolescents. American Journal of Psychiatry, 160, 1442-1452

Desikan, R., Segonne, F., Fischl, B., Quinn, B., Dickerson, B., Blacker, D.,... Buckner, R. (2006). An automated labeling system for subdividing the human cerebral cortex on MRI scans into gyral based regions of interest. Neuroimage, 31, 968-980

DeYoung, C. G. (2010). Mapping Personality Traits onto Brain Systems: BIS, BAS, FFFS and Beyond. European Journal of Personality 24, 404-407

Dinzeo, T. J. y Docherty, N. M. (2007). Normal personality characteristics in schizophrenia: a review of the literature involving the FFM. Journal of Nervous \&t Mental Disorders, 195, 421-429.

Downhill, J. E., Buchsbaum, M. S., Hazett, F. A., Barth, S., Rottman, S. L., Nunn, M. y Oksana, L. (2001). Temporal volumen determined by magnetic resonance spectroscopy Imaging in schizotipal personality disorder and schizophrenia. Schizophrenia Research, 30, 187-198

Durazzo, T. C. y Meyerhoff, D. J. (2007). Neurobiological and neurocognitive effects of chronic cigarette smoking and alcoholism. Frontiers in Bioscience, 12, 4079-4100.

Durazzo, T. C., Tosun, D., Buckley. S., Gazdzinski, S., Mon, A., Fryer, S. L.,... Meyerhoff, D. J. (2011). Cortical thickness, surface area, and volume of the brain reward system in alcohol dependence: relationships to relapse and extended abstinence. Alcoholism: Clinical and Experimental Research. 35, 1187-1200

Elkins, I. J., King, S. M., McGue, M., y lacono, W. G. (2006). Personality traits and the development of nicotine, alcohol, and illicit drug disorders: Prospective links from adolescence to young adulthood. Journal of Abnormal Psychology, 115, 26-39.

Everitt, B. J., Belin, D., Economidou, D., Pelloux, Y., Dalley, J. W. y Robbins, T. W. (2008). Review. Neural mechanisms underlying the vulnerability to develop compulsive drug-seeking habits and addiction. Philosophical Transactions of the Royal Society of London B Biological Sciences, 363, 3125-35

Everitt, B. J. y Robbins, T. W. (2005). Neural systems of reinforcement for drug addiction: from actions to habits to compulsion. Nature Neuroscience, 8, 1481-9

Ferguson, K. J., MacLullich, A. M., Marshall, I., Deary, I. J., Starr, J. M., Seckl, J. R.,...Wardlaw, J. M. (2002). Magnetic resonance spectroscopy and cognitive function in healthy elderly men. Brain, 125, 2743-2749. 
First, M. B., Spitzer, R. L., Gibbon, M. y Williams, J. B. (1999). Guía del usuario para la Entrevista Clínica Estructurada para los Trastornos del Eje I deI DSM-IV. Versión clínica. SCID-I. Barcelona: Masson.

First, M. B., Spitzer, R. L., Gibbon, M. y Williams, J. B. (1999). Entrevista Clínica Estructurada para los Trastornos de Personalidad del Eje II del DSM IV. Barcelona-Masson.

Gazdzinski, S., Durazzo, T. C., Mon, A. y Meyerhoff, D. J. (2010). Body Mass Index Is Associated With Brain Metabolite Levels in Alcohol Dependence: A Multimodal Magnetic Resonance Study. Alcoholism: Clinical and Experimental Research, 34, 2089-2096.

Gutiérrez-Zotes, J. A., Gutiérrez, F., Valero, J., Gallego, E., Baillés, E. y Torres, X. (2008). Structure of personality pathology in normal and clinical samples: Spanish validation of the DAPP-BO. Journal of Personality Disorders, 22, 389-404.

Hamakawa, H., Kato, T., Murashita, J. y Kato, N. (1998) Quantitative proton magnetic resonante spectroscopy of the basal ganglia in patients with affective disorders. European Archives of Psychiatry \& Clinical Neurosciences, 248, 53-5

Hays, W. L. (1981). Statistics (3rd ed). New York: Holt, Rinehart y Winston, 1981.

Helzer, J. E., Bucholz, K. K., y Gossop, M. (2008). A dimensional option for the diagnosis of substance dependence in DSM-V , en J. E. Helzer, $H$. C. Kraemer, R. F. Krueger, H. U. Wittchen, P. J. Sirovatka y D. A. Regier (Eds.), Dimensional approach in diagnostic classification. Refining the research agenda for DSM-V (pp. 19-34). Virginia: American Psychiatric Association.

Hernández, A., Gutiérrez, F., Valero, J., Gárriz, M., Labad, A., y GutiérrezZotes, J. A. (2009). Una alternativa genético-conductual a los trastornos de la personalidad: el modelo dimensional de Livesley. Actas Españolas de Psiquiatría, 37, 174-183.

Herrero, J. (2004). Alteraciones de la personalidad asociadas a las conductas adictivas. Influencia de la duración del consumo y sus implicaciones. Psyche, 13, 91-100.

Hopwood, C. J., Morey, L. C., Skodol, A. E., Stout, R. L., Yen, S. y Ansell, E. B. (2007). Five-factor model personality traits associated with alcohol-related diagnoses in a clinical sample. Journal of Studies on Alcohol and Drugs, 68, 455-460.

Joos, L., Goudriaan, A. E., Schmaal, L., De Witte, N. A., Van den Brink, W., Sabbe, B. G.,...Dom, G. (2013). The relationship between impulsivity and craving in alcohol dependent patients. Psychopharmacology, 226, 273-283

Jung, R. E., Gasparovic, C., Chavez, R. S., Caprihan, A. y Barrow, R. (2009). Imaging intelligence with proton magnetic resonance spectroscopy. Intelligence, 37, 192-198

Jung, R. E., Gasparovic, C., Chavez, R. S., Flores, R. A. y Smith, S. M. (2009). Biochemical support for the "threshold" theory of creativity: a magnetic resonance spectroscopy study. Journal of Neuroscience, $29,5319-5325$

Jung, R. E., Yeo, R. A., Love, T. M., Petropoulos, H. y Sibbitt, W. L. (2002). Biochemical markers of mood: a proton magnetic resonance spectroscopy study of normal human brain. Biological Psychiatry, $51,224-229$
Kalivas, P. W. y Volkow, N. D. (2005). The neural basis of addiction: A pathology of motivation and choice. American Journal of Psychiatry, 162, 1403-1413.

Krueger, R. F., y Tackett, J. L. (2003). Personality and psychopathology: Working toward the bigger picture. Journal of Personality Disorders, $17,109-128$.

Makris, N., Meyer, J. W., Bates, J. F., Yeterian, E. H., Kennedy, D. N. y Caviness, V. S. (1999). MRI-based topographic parcellation of human cerebral white matter and nuclei: II. Rationale and applications with systematics of cerebral connectivity. Neuroimage, 9, 18-45.

Mateos Agut, J. y Mateos Agut, M. (2005). Rasgos diferenciales del temperamento y el carácter en un grupo de alcohólicos vs población general. Adicciones 2005, 17, 325-335.

McFarland, K., Lapish, C. C. y Kalivas, P. W. (2003). Prefrontal glutamate release into the core of the nucleus accumbens mediates cocaineinduced reinstatement of drug-seeking behavior. Journal of Neuroscience, 23, 3531-3537.

McGue, M., Slutske, W. y lacono, W. G. (1999). Personality and substance use disorders: II. Alcoholism versus drug use disorders. Journal of Consulting and Clinical Psychology, 67, 394-404.

Mega, M. S. y Cummings, J. L. (1994). Frontal-subcortical circuits and neuropsychiatric disorders. Journal of Neuropsychiatry and Clinical Neurosciences, 6, 358-370.

Meyerhoff, D. J., Durazzo, T. C. y Ende, G. (2013). Chronic alcohol consumption, abstinence, and relapse: brain proton magnetic spectroscopy studies in animals and humans. Current Topics in Behavioral Neuroscience, 13, 511-40.

Omura, K., Constable, R. y Canli, T. (2005). Amygdala gray matter concentration is associated with extraversion and neuroticism. Neuroreport 16, 1905-1908

Oquendo, M. A., Baca-García, E., Graver, R., Morales, M., Montalvan, V. Y Mann, J. J. (2001). Spanish adaptation of the Barratt Impulsiveness Scale (BIS-11). European Journal of Psychiatry, 15, 147-55.

Paul, R., Grieve, S. M., Chaudary, B., Gordon, N., Lawrence, J., Cooper, N.,...Gordon, E. (2009). Relative contributions of the cerebellar vermis and prefrontal lobe volumes on cognitive function across the adult lifespan. Neurobiology of Aging, 30, 457-465.

Pfleiderer, B., Ohrmann, P., Suslow, T., Wolgast, M. y Gerlach, A. L. (2004). Nacetylaspartate levels of left frontal cortex are associated with verbal intelligence in women but not in men: a proton magnetic resonance spectroscopy study. Neuroscience 123, 1053-1058

Ready, R. E.,Watson, D., y Clark, L. A. (2006). Psychiatric patient- and informant-reported personality: Predicting concurrent and future behavior. Assessment, 9, 361-372

Ruiz, M. A., Pincus, A. L., y Schinka, J. A. (2008). Externalizing pathology and the five-factor model: A meta-analysis of personality traits associated with antisocial personality disorder, substance use disorder, and their co-occurrence. Journal of Personality Disorders, 22, 365-388.

Salazar-Fraile, J., Ripoll-Alandes, C. y Bobes J. (2010). Narcisismo manifiesto, narcisismo encubierto y trastornos de personalidad en una Unidad de Conductas Adictivas: validez predictiva de respuesta a tratamiento. Adicciones, 22, 107-112 
Schlaepfer, T. E., Lancaster, E., Heidbreder, R., Strain, E. C., Kosel, M., Fisch, H. U.,...Pearlson, G. D. (2006). Decreased frontal whitematter volume in chronic substance abuse. International Journal of Neuropsychopharmacology, 9, 147-153.

Schulte, T., Oberlin, B. G., Kareken, D. A., Marinkovic, K., Müller-Oehring, E. M., Meyerhoff, D. J.,...Tapert, S. (2012). How Acute and Chronic Alcohol Consumption Affects Brain Networks: Insights into Multimodal Neuroimaging. Alcoholism: Clinical and Experimental Research, 36, 2017-2027

Shedler, J. y Block, J. (1990). Adolescent drug use and psychological health. American Psychologist, 45, 612-630

Sher, K. J., Grekin, E. R., y Williams, N. A. (2005). The development of alcohol use disorders. Annual Review of Clinical Psychology, 1, 493523.

Soher, B. J., Young, K., Govindaraju, V. y Maudsley, A. A. (1998). Automated spectral analysis III: Application to in vivo proton MR spectroscopy and spectroscopic imaging. Magnetic Resonance in Medicine, 40, 822-831.

Spielberger, C. D., Gorsutch, R. I. y Lushene, R. (1982). Manual del cuestionario de ansiedad Estado/rasgo (STAl) (3a edición). Madrid; TEA.

Sullivan, E. V., Harding, A. J., Pentney, R. J., Dlugos, C. A., Martin, P. R., Parks, M. H.,...Pfefferbaum, A. (2003). Disruption of frontocerebellar circuitry and function in alcoholism. Alcoholism: Clinical and Experimental Research, 2003, 27:301-309.

Tessner, K. y Hill, S. (2010). Neural Circuitry Associated with Risk for Alcohol Use Disorders. Neuropsychology Review, 20, 1-20.

Thomson, A. D., Guerrini, I., Bell, D., Drummond, C., Duka, T., Field, M.,... Kopelman, M. (2012). Alcohol-Related Brain Damage: Report from a Medical Council on Alcohol Symposium. Alcohol and Alcoholism, 47, 84-91

Tibbo, P., Hanstock, C., Valiakalayil, A. y Allen, P. (2004). 3-T proton MRS investigation of glutamate and glutamine in adolescents at high genetic risk for schizophrenia. American Journal of Psychiatry, 161, 1116-1118

Van Elst, L. T., Thiel, T., Hesslinger, B., Lieb, K., Bohus, M., Henning, J.,... Ebert, D. (2001). Subtle prefrontal neuropathology in a pilot MRS study in patients with borderline personality disorders. Jorunal of Neuropsychiatry \& Clinical Neuroscience, 13, 511-4

Verdejo-García, A., Pérez-Garcia, M. y Bechara, A. (2006). Emotion, Decision-Making and Substance Dependence: A Somatic-Marker Model of Addiction. Current Neuropharmacology, 4, 17-31

Walter, M., Gerhard, U., Duersteler-MacFarland, K. M., Weijers, H. G., Boening, J. y Wiesbeck, G. A. (2006). Social factors but not stress-coping styles predict relapse in detoxified alcoholics. Neuropsychobiology, 54, 100-6 